OPEN ACCESS

Edited by:

Marco Scarci

San Gerardo Hospital, Italy

Reviewed by:

Nuria Maria Novoa,

University of Salamanca Health Care

Complex, Spain

Alper Toker,

West Virginia University, United States

${ }^{*}$ Correspondence:

Stephanie H. Chang

stephanie.chang@nyulangone.org

Specialty section:

This article was submitted to

Surgical Oncology,

a section of the journal

Frontiers in Surgery

Received: 02 February 2021

Accepted: 23 February 2021

Published: 07 April 2021

Citation:

Wang A, Chang SH, Kim EJ, Bessich JL, Sabari JK, Cooper B,

Geraci TC and Cerfolio RJ (2021)

Dynamic Management of Lung

Cancer Care During Surging

COVID-19. Front. Surg. 8:663364.

doi: 10.3389/fsurg.2021.663364

\section{Dynamic Management of Lung Cancer Care During Surging COVID-19}

\author{
Annie Wang ${ }^{1}$, Stephanie H. Chang ${ }^{2 *}$, Eric J. Kim ${ }^{3}$, Jamie L. Bessich ${ }^{4}$, Joshua K. Sabari ${ }^{4}$, \\ Benjamin Cooper ${ }^{5}$, Travis C. Geraci ${ }^{2}$ and Robert J. Cerfolio ${ }^{2}$
}

${ }^{1}$ Department of Surgery, New York University Langone Health, New York, NY, United States, ${ }^{2}$ Department of Cardiothoracic Surgery, New York University Langone Health, New York, NY, United States, ${ }^{3}$ New York University Grossman School of Medicine, New York, NY, United States, ${ }^{4}$ Department of Medicine, New York University Langone Health, New York, NY, United States, ${ }^{5}$ Department of Radiation Oncology, New York University Langone Health, New York, NY, United States

Management of patients with lung cancer continues to be challenging during the COVID-19 pandemic, due to the increased risk of complications in this subset of patients. During the COVID-19 surge in New York City, New York University Langone Health adopted triage strategies to help with care for lung cancer patients, with good surgical outcomes and no transmission of COVID-19 to patients or healthcare workers. Here, we will review current recommendations regarding screening and management of lung cancer patients during both a non-surge phase and surge phase of COVID-19.

Keywords: lung cancer, thoracic surgery, COVID-19, radiation oncology, screening

\section{INTRODUCTION}

The novel Coronavirus disease 2019 (COVID-19) is a respiratory viral infection caused by severe acute respiratory syndrome coronavirus 2 (SARS-Cov-2). Since first identification of SARS-CoV-2 in December 2019, COVID-19 emerged as a major global pandemic with over 98 million people diagnosed with COVID-19 and $>2$ million deaths worldwide as of January 2021 (1-3). The United States became the major epicenter for the pandemic, with 24 million patients diagnosed with COVID-19 and 408,000 deaths (1-3). Unfortunately, New York State, and in particular, New York City (NYC), has experienced the highest concentration of COVID-19 cases, with 1.2 million confirmed diagnoses and more than 41,000 deaths (1-3).

Early data, primarily from China, demonstrated that $15-20 \%$ of infected patients develop symptoms requiring hospitalization with a mortality rate of $\sim 1-4 \%(4-6)$. Patients with underlying medical co-morbidities, particularly pulmonary disease, diabetes, obesity, and heart disease have been demonstrated to be at a higher risk for morbidity and mortality (7-9). In patients with underlying malignancies, the mortality related to concomitant COVID-19 was notably higher at $\sim 20-40 \%$ (10-16). Lung cancer patients particularly have more severe COVID-19 disease, with an estimated mortality rate of $30-50 \%(11,17,18)$; this may be because lung tumor microenvironment can be modulated by SARS-CoV-2 virus, triggering a more severe cytokine response $(19,20)$. Lung cancer also creates immunosuppressive state that may predispose patients to COVID-19 and complications. Therefore, management of patients with lung cancer poses unique challenges during the SARS-COV2 pandemic.

COVID-19 cases continue to increase in number. While the vaccines promises to help slow the spread of COVID-19, new strains pose as another source for continued growth (21-23). It is imperative to restructure the clinical workflow and organization of hospitals to cope with the surge in the number of hospitalizations from COVID-19. 
TABLE 1 | Triage levels used to guide management strategies.

\begin{tabular}{lllll}
\hline $\begin{array}{l}\text { Triage } \\
\text { level }\end{array}$ & $\begin{array}{l}\text { Overall } \\
\text { transmission }\end{array}$ & $\begin{array}{l}\text { Local (city) } \\
\text { transmission }\end{array}$ & Hospital cases & $\begin{array}{l}\text { Hospital } \\
\text { capacity }\end{array}$ \\
\hline Level 1 & Present & Minimal/None & Minimal/None & Available beds \\
Level 2 & Present & Multiple cases & Minimal/None & Available beds \\
Level 3 & Present & Multiple cases & Multiple cases & Available beds \\
Level 4 & Present & Multiple cases & Multiple cases & Surge units full \\
\hline
\end{tabular}

In this review article, we will discuss lung cancer management during COVID-19 pandemic and present our current institutional practice and outcomes. The work-up and management of patients depends on rate of new infections and hospitalizations for COVID-19. At NYU Langone Health (NYULH), we follow a tiered approach based on transmission rate. Transmission of COVID-19 outside of New York City (NYC), with little or no cases in NYC, and no cases at NYULH is deemed level 1 . Level 2 is when there are no local transmission, but multiple cases in NYC and few NYULH cases. Transmission in NYULH is level 3, while level 4 is when NYULH surge units are full (Table 1).

\section{MANAGEMENT DURING NON-SURGE PHASE WITH ADEQUATE HOSPITAL CAPACITY (LEVEL 1 AND 2)}

If the city and hospital are not in a surge phase of COVID19 outbreak and have available resources, standard criteria for lung cancer work-up and management should be followed with several added precautions. Patients with lung cancer require frequent clinic visits with multiple specialists, and necessitate diagnostic work up with laboratory blood draws, imaging studies, pulmonary function tests (PFTs), bronchoscopies and transthoracic needle biopsies, radiation treatments, antineoplastic infusion treatments and hospital admissions for procedures. These frequent visits increased both the patient risk of exposure to COVID-19, as well as increased risk of exposure to healthcare providers taking care of lung cancer patients (Figure 1).

All patients presenting for in-person appointments should be contacted $24-48 \mathrm{~h}$ prior to the appointment and screened for flu-like symptoms: cough, dyspnea, fevers, chills, and loss of smell or taste. If patients have any symptoms during the telephone screening, the appointments are canceled. If patients required further evaluation of symptoms, they should be triaged for COVID-19 testing and referred to emergency room when appropriate. If patients test positive for COVID-19, in-person clinic and infusion appointments were deferred until patients were asymptomatic for a minimum of 14 days. Those requiring more immediate attention for their cancer management should be admitted for inpatient management.

All healthcare providers must wear N95 masks, eye shield, and gloves for patient contact. Face masks or coverings should be worn by all visitors and patients, regardless of their symptoms.
Patient entry into the cancer center should restricted to one point of entry and access was controlled by a team of medical assistants and a nurse. COVID-19 clinical screening questions and COVID19 test results should documented prior to registration and inperson clinic visit. If a patient screens positive with suspicion for COVID-19, the patient should be shifted to an isolation area. The patient's team would perform a clinical assessment in the isolation area and determine the appropriate action. If patients needed to be tested for COVID-19, the clinic visit and treatments were delayed until the results of the test were available.

Previously, there was concerns about bronchoscopy as a super-spreading event (24), but our published data have shown no transmission during bronchoscopy of active COVID-19 patients when appropriate precautions are taken (25). This lack of transmission has also been reproduced by other institutions $(26,27)$. Thus, normal preoperative bronchoscopy with biopsy or endobronchial ultrasound (EBUS) for staging should continue if patients test negative for COVID-19 within 3 days of their procedure. Similarly, PFTs should also be obtained in routine fashion if the patient has a negative test within 3 days. All treatment decisions regarding surgery, adjuvant and neoadjuvant therapy, and radiation therapy should follow normal guidelines, with negative COVID-19 testing within 3 days of any surgical procedure. All surgical procedures should be performed with operating room staff wearing N95 masks, eye shield, and gloves for patient contact. Post-operative management, including management of pneumothoraces and air leaks, should follow standard practice patterns prior to the COVID-19 pandemic.

\section{MANAGEMENT DURING SURGE PHASE OR WHEN LIMITED HOSPITAL CAPACITY (LEVEL 3 AND 4) \\ Changes to Initial Procedures for Suspected or Diagnosed Lung Cancer Patients}

Patients with suspected diagnosis or newly diagnosed lung cancer require a variety of imaging modalities, diagnostic biopsies, and staging procedures. Following announcement of COVID-19 as a national emergency in March 2020, guidelines were issued by the American Association of Bronchology and Interventional Pulmonology and the American College of Chest Physicians (24), which includes the following measures to prioritize patients for diagnostic work up of known or suspected lung cancer.

All cases considered for bronchoscopy should be discussed at our multi-disciplinary thoracic oncology conference (MDTOC) to determine the optimal course for diagnosis and staging from the perspective of both the patient and the lung cancer team. Staging endobronchial ultrasounds were discouraged in patients without radiographic lymphadenopathy. In these patients, mediastinal and hilar staging via EBUS guided transbronchial needle aspiration (TBNA) was deferred, given the relatively low likelihood of macro- or microscopic metastases in regional lymph nodes in this setting. This was particularly valid if the standardized uptake value (SUV) of the primary lesion in the lung parenchyma was relatively low (e.g., <5-7) on 


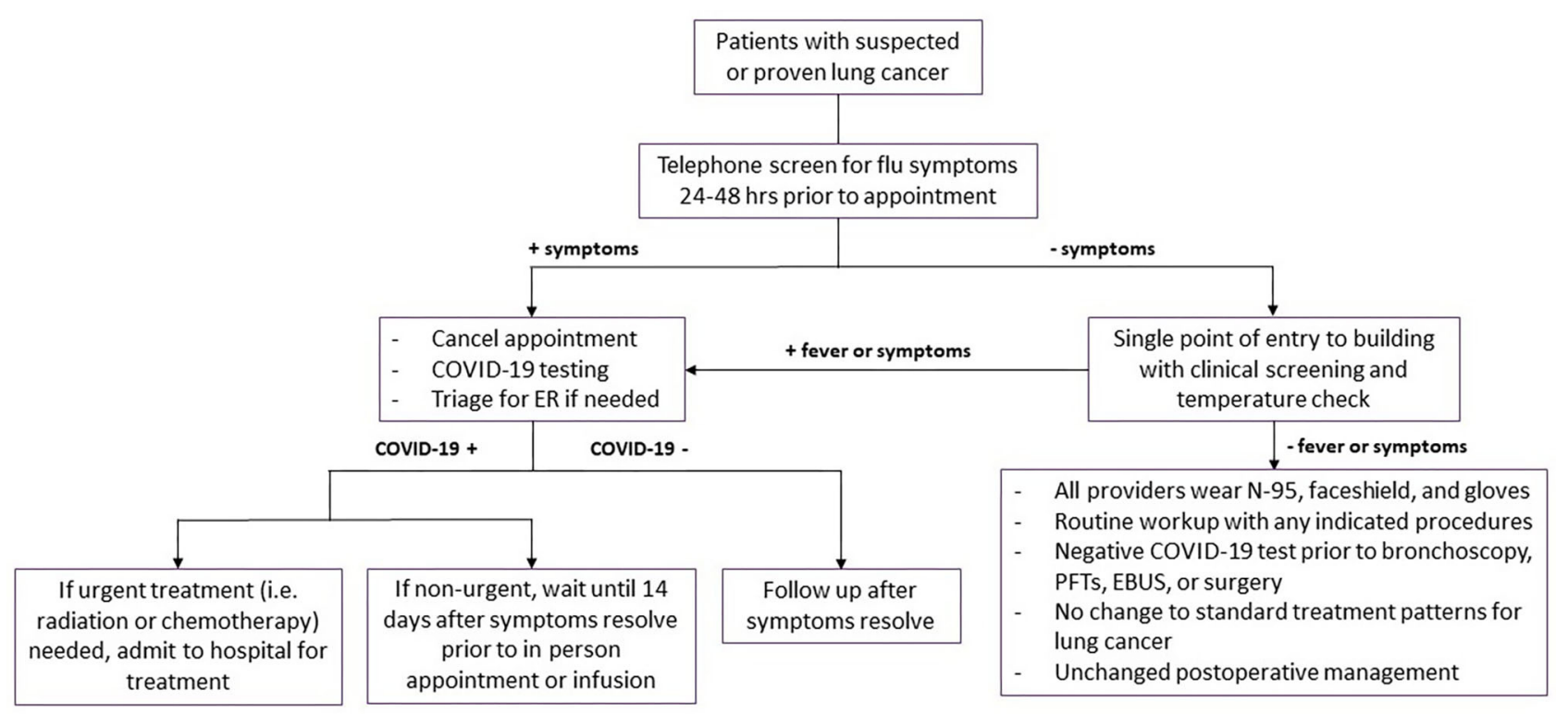

FIGURE 1 | Management of patients during Level 1 and Level 2 phases of transmission. Flowchart demonstrating management and workup strategies for patients with suspected or diagnosed lung cancer during level 1 and level 2 phases of transmission. Symptoms include fevers, chills, cough, dyspnea, and loss of smell or taste. COVID-19, coronavirus disease 2019; ER, emergency room; PFTs, pulmonary function tests; EBUS, endobronchial ultrasound.

positron emission tomography (PET). Conversely, patients with compelling evidence of malignant adenopathy were managed without tissue confirmation (Figure 2).

Diagnostic biopsies of peripheral lung nodules or masses in the absence of significant lymphadenopathy were performed by transthoracic needle aspiration by interventional radiology. This was considered a lower risk procedure for aerosolization of the SARS CoV-2 virus than navigational or robotic bronchoscopy for biopsy of these lesions.

For patients with confirmed peripheral lung cancer on prior biopsy who were being considered for definitive stereotactic body radiotherapy (SBRT) by radiation oncology or percutaneous cryoablation by Thoracic Radiology, we deferred staging EBUS TBNA of hilar and mediastinal nodes in those without significant adenopathy on cross sectional imaging or evidence of mediastinal or hilar FDG avidity on PET CT scan.

All patients with suspected new or recurrent diagnoses of lung cancer recommended at MDTOC to undergo EBUS TBNA should undergo SARS-CoV-2 nasopharyngeal swab RT-PCR testing prior to the procedure. Patients with negative SARS-CoV2 testing could proceed to bronchoscopy with the interventional pulmonology team, with strict precautions within the operating room. These included full use of personal protective equipment by all OR staff (N95 masks, face shields, and impermeable gowns), as well as ensuring that only essential staff were present in the operating room for anesthesia induction and securing the airway. Patients scheduled to undergo diagnostic and staging bronchoscopy who had positive SARS-CoV-2 RT PCR testing prior to the procedure were quarantined for 2 weeks and re-tested prior to proceeding with the endoscopic procedure.
For those lung cancer patients presenting with symptomatic central airway obstruction (persistent cough, dyspnea, hemoptysis, hypoxemia, post-obstructive atelectasis, or pneumonia), therapeutic bronchoscopy (rigid bronchoscopic debulking, thermal ablation, airway stenting) should be performed as clinically indicated regardless of SARS-CoV-2 status. SARS-CoV-2 testing should still be performed within 3 days of the planned procedure for risk stratification and preparation for safest possible conditions for the interventional pulmonologists and the OR staff (Figure 3).

We recommend continuing diagnostic and therapeutic pleural procedures in patients who are candidates for systemic antineoplastic therapy. Proceduralists should adhere to strict contact precautions with face shields, N95 masks, impermeable gowns, and sterile gloves. For patients symptomatic from known malignant pleural effusions, we recommend placement of indwelling pleural catheter (IPC) insertion. IPC placement is thought to be associated with lower risk of SARS-CoV2 aerosolization than talc insufflation via video assisted thoracoscopic surgery (VATS) and could be performed in an expedited fashion on an outpatient basis. When patients had IPC insertions, a family member was remotely trained and counseled on safe and proper drainage of the IPC to obviate requirements for home visiting nurse encounters to minimize risk of COVID19 exposure.

\section{Changes to Curative Surgical Management of Lung Cancer}

All patients should be discussed in MDTOC, with decisions regarding surgical resection determined by the growth rate and 


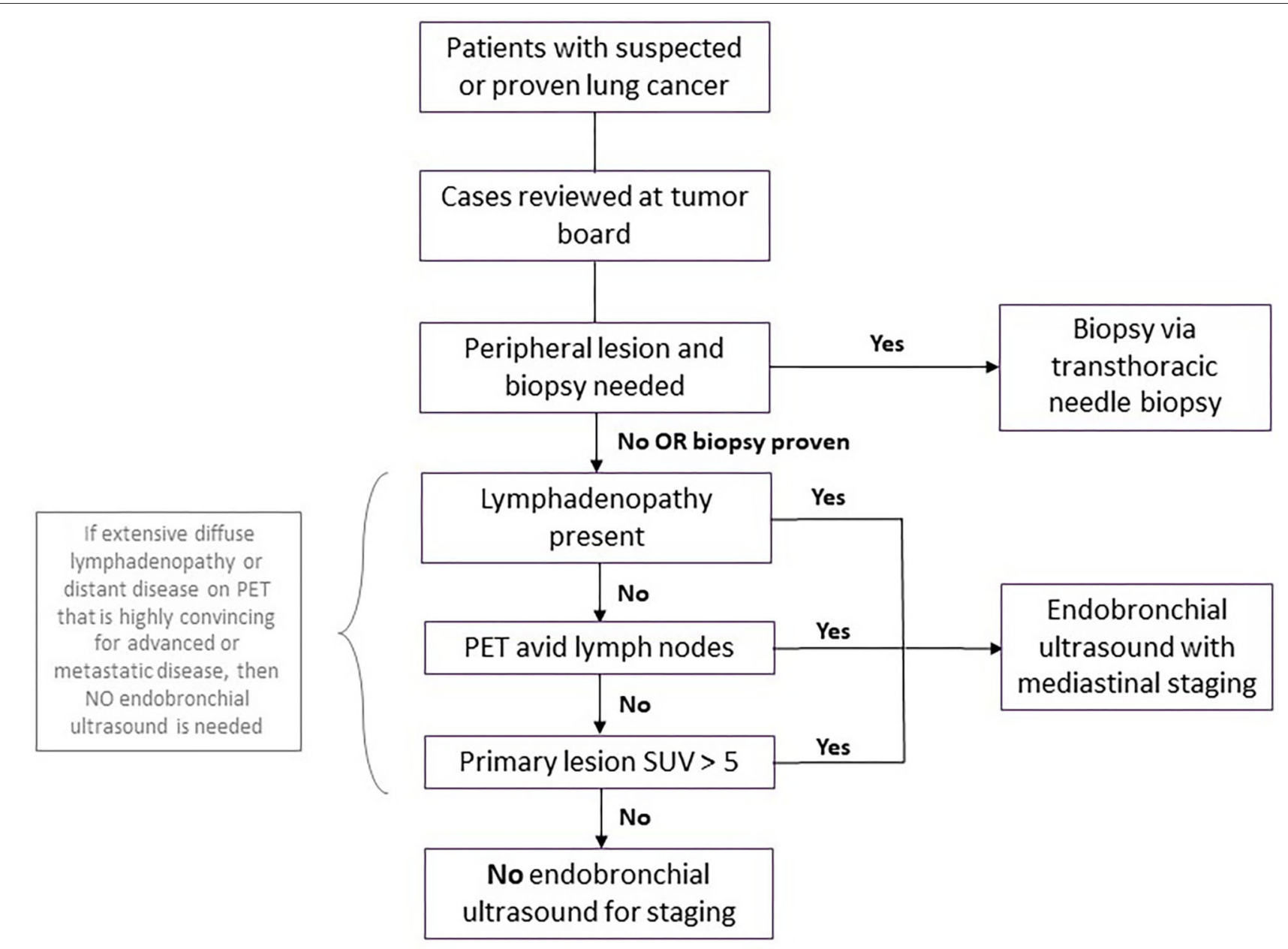

FIGURE 2 | Workup for patients with suspected or proven lung cancer during surge phase or limited hospital capacity. Flowchart demonstrating workup for patients with suspected or proven lung cancer during surge phase or limited hospital capacity. A coronavirus disease 2019 (COVID-19) test should be performed prior to the biopsy or endobronchial ultrasound, with the procedure deferred for any patient with COVID-19 until the patient is negative. PET, positive electron tomography; SUV, standard uptake value.

FDG avidity of the cancer in serial imaging studies, patient clinical factors and the co-morbidities including COVID-19 status. Patients with a solid or $>50 \%$ solid nodule $>2 \mathrm{~cm}, \mathrm{SUV}_{\max }$ $>2.5$, or change on short interval CT scan were deemed higher risk for progression of disease with delay in care and prioritized for resection. Patients on neoadjuvant trials should also undergo resection during this surge phase if there are no other contraindications. The guidelines used by our multidisciplinary group at NYULH echoed the recommendations of the American College of Surgeons and the Thoracic Surgery Outcomes Research Network $(28,29)$. Specifically, surgery was essentially restricted to patents whose survivorship was likely to be compromised by a surgical delay of 3 months. Conditions for early surgery included: (1) solid or predominantly solid (>50\%) lung cancer or presumed lung cancer $>2 \mathrm{~cm}$, clinical node negative, (2) node positive lung cancer, and (3) post-induction therapy cancers. Moreover, staging to start treatment (EBUS, mediastinoscopy, diagnostic VATS) for pleural dissemination was also performed. Generally, surgery was deferred for an estimated 3 months for predominantly ground glass nodules or cancers, solid nodules or lung cancer $<2 \mathrm{~cm}$, and radiographic/biopsy signs of an indolent tumor (carcinoid, slowly enlarging nodule) (Figure 4).

The outcomes of these criteria applied at the peak of COVID19 pandemic to 21 patients at NYULH were retrospectively reviewed (30), and there were no major complications, 5 minor complications and average length of stay of 2 days. Twenty-four patients deferred surgery due to patient preference and only one of these patients progressed from T3N0 to T4N0 disease. Notably, none of the nine healthcare providers involved in the surgical management of these patients contracted COVID-19.

Given the high risk to patients after lung resection, especially cancer patients that were immunocompromised and at greater risk of COVID-19 complications, surgical interventions for 


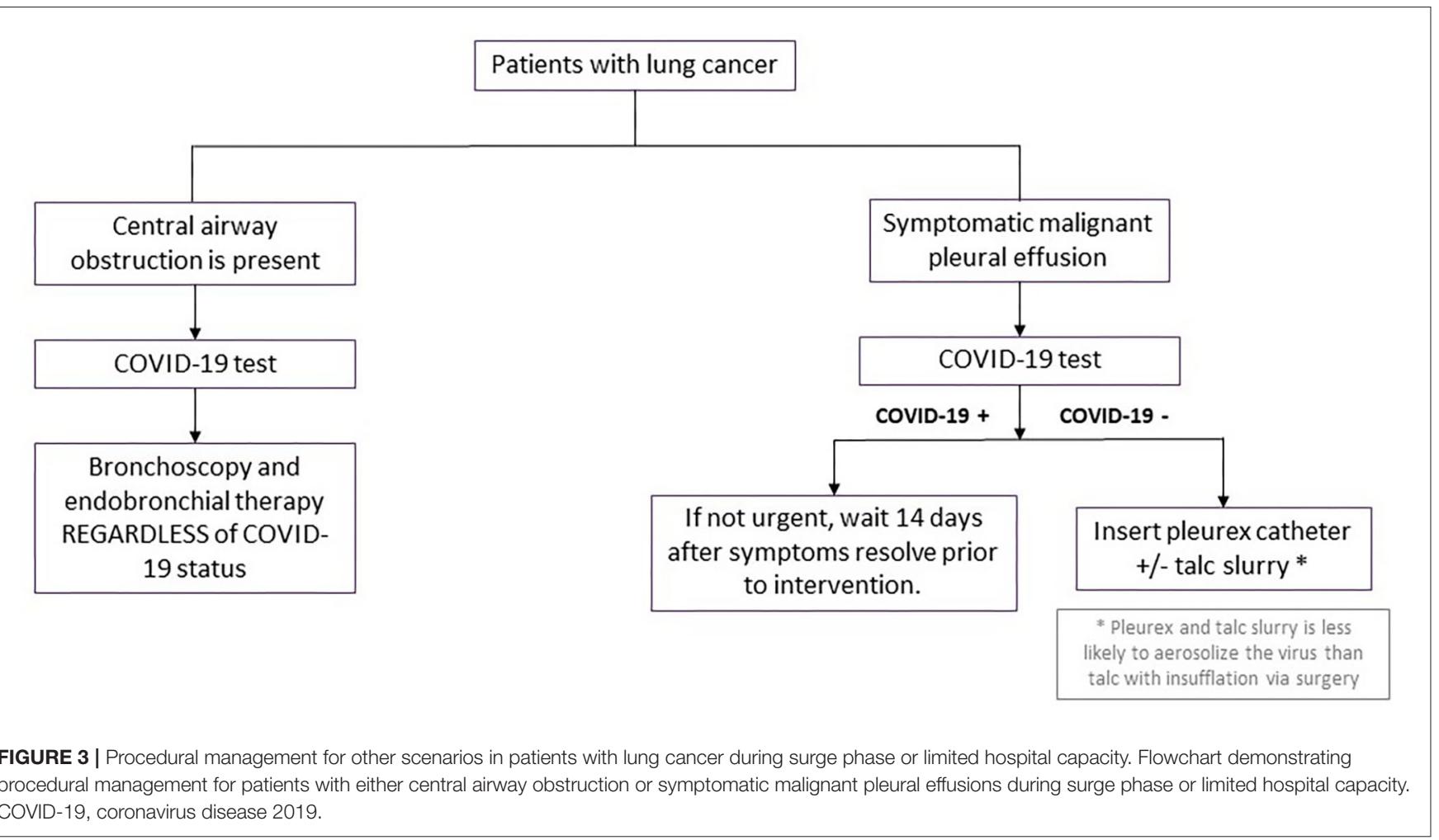

stage 1 NSCLC should be deferred when clinically appropriate. This increased selection process will also decrease unnecessary exposure of healthcare personnel to potential asymptomatic COVID-19 patients with a false negative test secondary to airway management. Pulmonary resections for lung cancer require endotracheal intubation, as well as bronchoscopy for double lumen endotracheal tube placement. No report has described increased rate of COVID-19 transmission from thoracic surgery, regardless of exposure type, compared to other types of surgery.

All physicians and operating room personnel should screened daily for fever or development of other COVID-19 symptoms. Any provider who cared for patients positive for COVID-19 should undergo RT-PCR testing every month. Symptomatic health care providers should be tested for COVID-19. If positive, the provider should be quarantined for 2 weeks; if negative, the provider quarantined until asymptomatic for 5 days. Patients should also undergo COVID-19 RT-PCR testing within $72 \mathrm{~h}$ of the operation. If positive, we recommend 2 week quarantine followed by re-testing. During our experience from March 13th to May 4th, 2020, one (5\%) of 21 patients was asymptomatic and found to be COVID-19 positive prior to surgery (30). Surgery was performed once the patient had a negative COVID19 test. Regardless of the negative test, anesthesiologists should employ heightened intubation precautions. During intubation and bronchoscopy to position the endotracheal tube, all nonnecessary personnel should wait outside the operating room. All surgical cases were performed through a minimally invasive approach (robotic or video-assisted) with the goal of safe expedited discharge for outpatient follow-up to minimize risk of in-hospital viral transmission.

Standard post-operative care regarding chest tube management and discharge criteria should be used, including specific precautions regarding superspreading procedures such as extubation, and peri-operative respiratory therapy such as nebulized bronchodilators, incentive spirometry, high-flow nasal cannula oxygen and non-invasive ventilation. All in-hospital care was provided on specific COVID free operating rooms, ICUs and floors. No patients could have visitors during their hospital stay and follow up after resection was done using telehealth visits. Patients with T1/T2 N0 NSCLC were reviewed in the multi-disciplinary and if stereotactic radiation was an option, it should be recommended and discussed with patients. In patients for whom surgery was delayed, consideration was given for neoadjuvant systemic chemotherapy.

\section{Changes to Radiation Therapy for Lung Cancer}

Several changes in the clinical workflow should be made to meet adequate social distancing guidelines for lung cancer patients needing radiation. All follow-ups that do not require a physical exam should be changed to a virtual telemedicine platform. Most patients with stage I and II lung cancer considered for stereotactic body radiotherapy (SBRT) are elderly with poor lung function and other comorbidities that preclude surgical management; therefore, SBRT should be post-poned as appropriate during surge phase to minimize 


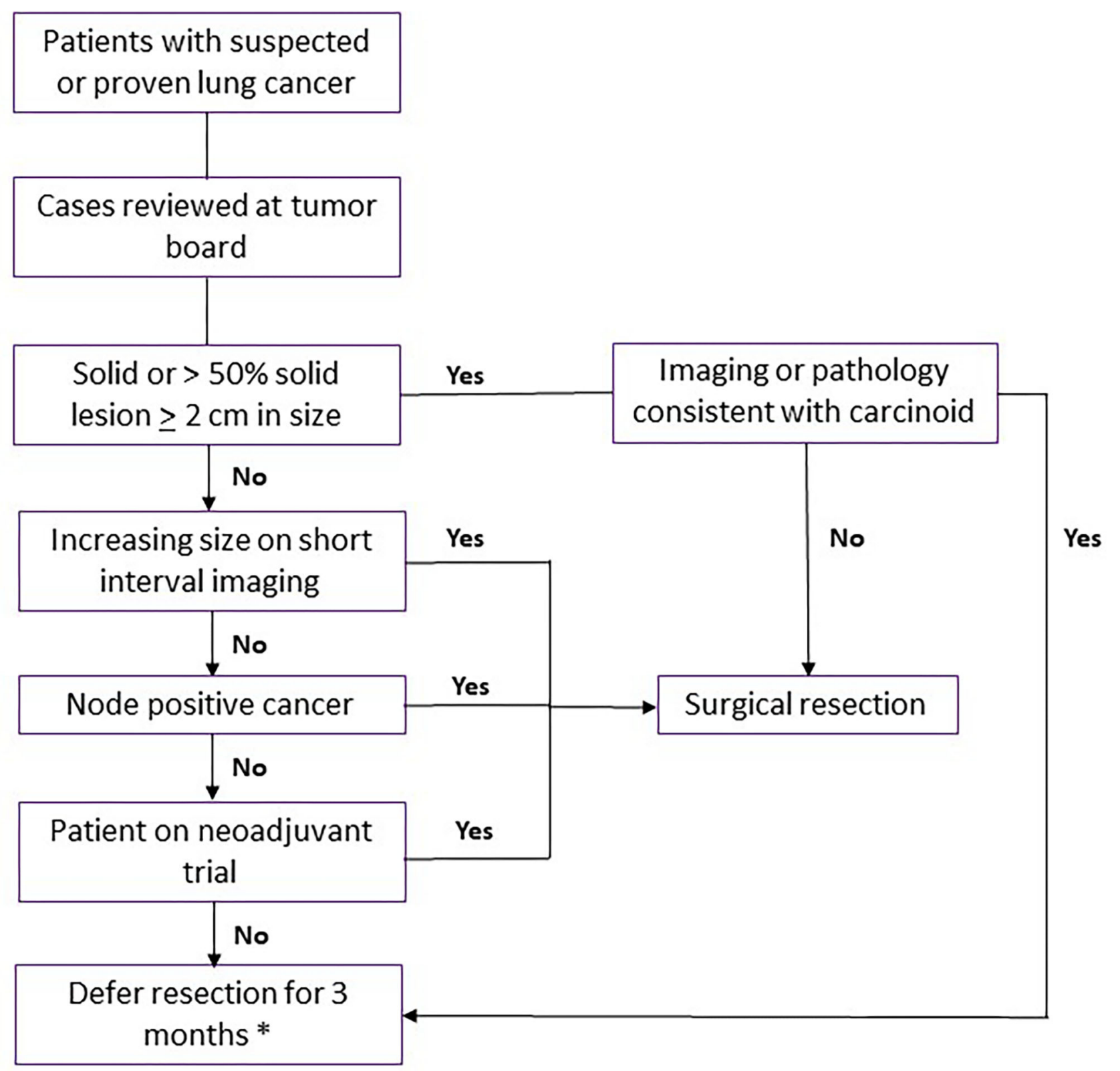

* T1/T2 N0 deferred patients can be evaluated for SBRT

* Patients with more advanced stage disease can be evaluated for neoadjuvant treatment

FIGURE 4 | Guidelines for curative surgical management of lung cancer during surge phase or limited hospital capacity. Flowchart demonstrating criteria for patients with suspected or diagnosed lung cancer who should undergo curative surgical resection, vs. waiting or consideration for another intervention. SBRT, stereotactic body radiation therapy.

COVID-19 infection risk (31). Furthermore, we considered single fraction SBRT based on encouraging results from RTOG 0915 which demonstrated comparable local tumor control with $34 \mathrm{~Gy}$ in a single fraction vs. $48 \mathrm{~Gy}$ in four consecutive daily fractions (32). No restrictions need to be imposed on curative intent concurrent or sequential chemoradiation treatments for patients with locally advanced small cell lung cancer (SCLC) and NSCLC.

Given the lack of level I evidence and controversy surrounding post-operative radiotherapy for patents with fully resected N2 
lung cancer (33), we recommend against adjuvant radiotherapy. Similarly, despite level I evidence supporting consolidative thoracic radiotherapy for patients with extensive stage small cell lung cancer (SCLC) (28), the lack of inclusion of thoracic RT in the IMPOWER 133 (34) and CASPIAN (35) studies combined with the poor prognosis and often compromised lung function of these patients led us to discontinue offering RT in this context. We also do not offer prophylactic cranial irradiation for patients with SCLC during the surge phase. Palliative radiotherapy was delivered with a single fraction for bone metastases when possible given similar initial efficacy to more protracted courses (36). Hypofractionation (17Gy in two fractions) was considered for patients requiring urgent palliative thoracic radiotherapy (37).

All patients should be evaluated for adequate pulmonary function, though this may be challenging as pulmonary function labs may be closed due to infection transmission concerns related to COVID-19. If recent pulmonary function tests are unavailable, we recommend clinical estimation of the pulmonary function using prior pulmonary function tests if available, baseline oxygen saturation, virtual walk desaturation testing and stair challenge. The standard radiation treatment time slots were increased from 15 to $20 \mathrm{~min}$ to minimize waiting room time as much as possible. All patients should masked, screened for COVID-19 symptoms, checked for elevated temperature, and maintained social distancing protocols. COVID-19 testing should be performed on all patients prior to CT simulation. Patients with active COVID-19 infection were treated with

\section{REFERENCES}

1. Dong E, Du H, Gardner L. An interactive web-based dashboard to track COVID-19 in real time. Lancet Infect Dis. (2020) 20:533-4. doi: 10.1016/S1473-3099(20)30120-1

2. Nuzzo J, Moss B, Kahn J, Rutkow L, Applied Physics Laboratory, Bauer B, et al. Johns Hopkins Coronavirus Resource Center. Johns Hopkins University (2021). Available online at: coronavirus.jhu.edu/us-map (accessed January 20, 2021).

3. CDC COVID Data Tracker. Centers for Disease Control and Prevention. (2021). Available online at: covid.cdc.gov/covid-data-tracker (accessed January 22, 2021).

4. Guan WJ, Ni ZY, Hu Y, Liang WH, Ou CQ, He JX, et al. Clinical characteristics of coronavirus disease 2019 in China. N Engl J Med. (2020) 382:1708-20. doi: 10.1056/NEJMoa2002032

5. Wu Z, McGoogan JM. Characteristics of and important lessons from the coronavirus disease 2019 (COVID-19) outbreak in China: summary of a report of 72314 cases from the Chinese Center for Disease Control and Prevention. JAMA. (2020) 323:1239-42. doi: 10.1001/jama.2020. 2648

6. Arneson D, Elliott M, Mosenia A, Oskotsky B, Solodar S, Vashisht $\mathrm{R}$, et al. CovidCounties is an interactive real time tracker of the COVID19 pandemic at the level of US counties. Sci. data. (2020) 7:405. doi: 10.1038/s41597-020-00731-8

7. Petrilli CM, Jones SA, Yang J, Rajagopalan H, O’Donnell L, Chernyak Y, et al. Factors associated with hospital admission and critical illness among 5279 people with coronavirus disease 2019 in New York City: prospective cohort study. BMJ. (2020) 369:m1966. doi: 10.1136/bmj.m1966

8. Richardson S, Hirsch JS, Narasimhan M, Crawford JM, McGinn T, Davidson KW, et al. Presenting characteristics, comorbidities, and outcomes among 5700 patients hospitalized with COVID-19 in the New York City Area. JAMA. (2020) 323:2052-9. doi: 10.1001/jama.2020.6775

9. Stefan N, Birkenfeld AL, Schulze MB, Ludwig DS. Obesity and impaired metabolic health in patients with COVID-19. Nat Rev Endocrinol. (2020) 16:341-2. doi: 10.1038/s41574-020-0364-6 radiation therapy if the initiation or continuation of such therapy was deemed essential following MDTOC review. Such patients were typically scheduled for last patient of the day treatments followed by terminal room and facility cleans. With these protocols in place, we were able to continue offering radiotherapy without significant staff shortages necessitating forced patient delays throughout the pandemic.

\section{CONCLUSIONS}

Management is different based on whether the hospital system is in surge phase and the capacity of the hospital. We have proven that lung cancer surgery can be safely performed in a hospital with census saturated with COVID-19 patients. Furthermore, we have had providers care for both COVID-19 patients and non-COVID-19 lung cancer patients, with a $0 \%$ transmission rate. It is not feasible to have dedicated non-COVID centers for oncologic surgery as it decreases available resources in a pandemic; therefore, the recommendations summarized in this review can guide cancer centers to continue to provide lung cancer care during COVID-19 pandemic with minimal added risk to patients and healthcare providers.

\section{AUTHOR CONTRIBUTIONS}

All authors listed have made a substantial, direct and intellectual contribution to the work, and approved it for publication.

10. Liang W, Guan W, Chen R, Wang W, Li J, Xu K, et al. Cancer patients in SARS-CoV-2 infection: a nationwide analysis in China. Lancet Oncol. (2020) 21:335-7. doi: 10.1016/S1470-2045(20)30096-6

11. Mehta V, Goel S, Kabarriti R, Cole D, Goldfinger M, Acuna-Villaorduna $\mathrm{A}$, et al. Case fatality rate of cancer patients with COVID-19 in a New York hospital system. Cancer Disc. (2020) 10:935-41. doi: 10.1158/2159-8290.CD-20-0516

12. Erdal GS, Polat O, Erdem GU, Korkusuz R, Hindilerden F, Yilmaz M, et al. The mortality rate of COVID-19 was high in cancer patients: a retrospective singlecenter study. Int J Clin Oncol. (2021) 1-9. doi: 10.1007/s10147-021-01863-6. [Epub ahead of print].

13. Cai Y, Hao Z, Gao Y, Ping W, Wang Q, Peng S, et al. Coronavirus disease 2019 in the perioperative period of lung resection: a brief report from a single thoracic surgery department in Wuhan, People's Republic of China. J Thorac Oncol. (2020) 15:1065-72. doi: 10.1016/j.jtho.2020.04.003

14. Aminian A, Safari S, Razeghian-Jahromi A, Ghorbani M, Delaney CP. COVID-19 outbreak and surgical practice: unexpected fatality in perioperative period. Ann Surg. (2020) 272:e27-9. doi: 10.1097/SLA.0000000000003925

15. Li YK, Peng S, Li LQ, Wang Q, Ping W, Zhang N, et al. Clinical and transmission characteristics of Covid-19 - A retrospective study of 25 cases from a single thoracic surgery department. Curr Med Sci. (2020) 40:295-300. doi: 10.1007/s11596-020-2176-2

16. Gonfiotti A, Gatteschi L, Salvicchi A, Bongiolatti S, Lavorini F, Voltolini L. Clinical courses and outcomes of five patients with primary lung cancer surgically treated while affected by Severe acute respiratory syndrome coronavirus 2. Eur J Cardiothorac Surg. (2020) 58:598-604. doi: 10.1093/ejcts/ezaa233

17. Peravali M, Joshi I, Ahn J, Kim C. A systematic review and meta-analysis of clinical characteristics and outcomes in lung cancer patients with COVID19. JTO Clin Res Rep. (2021) 100141. doi: 10.1016/j.jtocrr.2020.100141. [Epub ahead of print].

18. Garassino MC, Whisenant JG, Huang LC, Trama A, Torri V, Agustoni F, et al. COVID-19 in patients with thoracic malignancies (TERAVOLT): first 
results of an international, registry-based, cohort study. Lancet Oncol. (2020) 21:914-22. doi: 10.1016/S1470-2045(20)30314-4

19. Malkani N, Rashid MU. SARS-COV-2 infection and lung tumor microenvironment. Mol Biol Rep. (2021) 1-10. doi: 10.1007/s11033-021-06149-8. [Epub ahead of print].

20. Ahmad S, Manzoor S, Siddiqui S, Mariappan N, Zafar I, Ahmad A, et al. Epigenetic underpinnings of inflammation: Connecting the dots between pulmonary diseases, lung cancer and COVID-19. Semin Cancer Biol. (2021). doi: 10.1016/j.semcancer.2021.01.003. [Epub ahead of print].

21. Moghadas SM, Vilches TN, Zhang K, Wells CR, Shoukat A, Singer BH, et al. The impact of vaccination on COVID-19 outbreaks in the United States. Clin Infect Dis. (2021). doi: 10.1093/cid/ciab079. [Epub ahead of print].

22. Muik A, Wallisch AK, Sänger B, Swanson KA, Mühl J, Chen W, et al. Neutralization of SARS-CoV-2 lineage B.1.1.7 pseudovirus by BNT162b2 vaccine-elicited human sera. Science. (2021). doi: 10.1101/2021.01.18.426984. [Epub ahead of print].

23. Wibmer CK, Ayres F, Hermanus T, Madzivhandila M, Kgagudi P, Lambson BE, et al. SARS-CoV-2 501Y.V2 escapes neutralization by South African COVID-19 donor plasma. Nat Med. (2021). doi: 10.1101/2021.01.18.427166. [Epub ahead of print].

24. Wahidi MM, Shojaee S, Lamb CR, Ost D, Maldonado F, Eapen G, et al. The use of bronchoscopy during the coronavirus disease 2019 Pandemic: CHEST/AABIP Guideline and Expert Panel Report. Chest. (2020) 158:126881. doi: $10.1016 /$ j.chest.2020.04.036

25. Chang SH, Jiang J, Kon ZN, Williams DM, Geraci TC, Smith DE, et al. Safety and efficacy of bronchoscopy in critically Ill patients with coronavirus disease 2019. Chest. (2020). doi: 10.1016/j.chest.2020.09.263. [Epub ahead of print].

26. Gao CA, Bailey JI, Walter JM, Coleman JM, Malsin ES, Argento AC, et al. Bronchoscopy on intubated COVID-19 patients is associated with low infectious risk to operators. Ann Am Thorac Soc. (2021). doi: 10.1513/AnnalsATS.202009-1225RL. [Epub ahead of print].

27. Lammers MJW, Lea J, Westerberg BD. Guidance for otolaryngology health care workers performing aerosol generating medical procedures during the COVID-19 pandemic. J Otolaryngol. (2020) 49:36. doi: 10.1186/s40463-020-00429-2

28. Likhitsup A, Parikh ND. Economic implications of hepatocellular carcinoma surveillance and treatment: a guide for clinicians. Pharmacoeconomics. (2020) 38:5-24. doi: 10.1007/s40273-019-00839-9

29. Antonoff M, Backhus L, Boffa DJ, Broderick SR, Brown LM, Carrott P, et al. COVID-19 guidance for triage of operations for thoracic malignancies: a consensus statement from thoracic surgery outcomes research network. Ann Thorac Surg. (2020) 110:692-6. doi: 10.1016/j.athoracsur.2020.03.005

30. Chang SH, Zervos M, Kent A, Chachoua A, Bizekis C, Pass H, et al. Safety of patients and providers in lung cancer surgery during the
COVID-19 pandemic. Eur J Cardio Thorac Surg. (2020) 58:1222-7. doi: 10.1093/ejcts/ezaa332

31. Guan WJ, Liang WH, Zhao Y, Liang HR, Chen ZS, Li YM, et al. Comorbidity and its impact on 1590 patients with COVID-19 in China: a nationwide analysis. Eur Respir J. (2020) 55: 2000547. doi: 10.1183/13993003.00547-2020

32. Videtic GM, Paulus R, Singh AK, Chang JY, Parker W, Olivier KR, et al. Long-term follow-up on NRG oncology RTOG 0915 (NCCTG N0927): a randomized phase 2 study comparing 2 stereotactic body radiation therapy schedules for medically inoperable patients with stage i peripheral nonsmall cell lung cancer. Int J Radiat Oncol Biol Phys. (2019) 103:1077-84. doi: 10.1016/j.ijrobp.2018.11.051

33. Wu SP, Shaikh F, Cerfolio R, Cooper BT. PORT in properly selected patients with completely resected non-small cell lung cancer should not be quickly dismissed. Ann Thorac Surg. (2019) 107:1585-6. doi: 10.1016/j.athoracsur.2018.09.071

34. Horn L, Mansfield AS, Szczesna A, Havel L, Krzakowski M, Hochmair MJ, et al. First-Line atezolizumab plus chemotherapy in extensive-stage small-cell lung cancer. N Engl J Med. (2018) 379:2220-9. doi: 10.1056/NEJMoa1809064

35. Paz-Ares L, Dvorkin M, Chen Y, Reinmuth N, Hotta K, Trukhin D, et al. Durvalumab plus platinum-etoposide versus platinum-etoposide in first-line treatment of extensive-stage small-cell lung cancer (CASPIAN): a randomised, controlled, open-label, phase 3 trial. Lancet. (2019) 394:1929-39. doi: 10.1016/S0140-6736(19)32222-6

36. Rich SE, Chow R, Raman S, Liang Zeng K, Lutz S, Lam H, et al. Update of the systematic review of palliative radiation therapy fractionation for bone metastases. Radiother Oncol. (2018) 126:547-57. doi: 10.1016/j.radonc.2018.01.003

37. Sundstrom S, Bremnes R, Aasebo U, Aamdal S, Hatlevoll R, Brunsvig P, et al. Hypofractionated palliative radiotherapy (17 Gy per two fractions) in advanced non-small-cell lung carcinoma is comparable to standard fractionation for symptom control and survival: a national phase III trial. $J$ Clin Oncol. (2004) 22:801-10. doi: 10.1200/JCO.2004.06.123

Conflict of Interest: The authors declare that the research was conducted in the absence of any commercial or financial relationships that could be construed as a potential conflict of interest.

Copyright (c) 2021 Wang, Chang, Kim, Bessich, Sabari, Cooper, Geraci and Cerfolio. This is an open-access article distributed under the terms of the Creative Commons Attribution License (CC BY). The use, distribution or reproduction in other forums is permitted, provided the original author(s) and the copyright owner(s) are credited and that the original publication in this journal is cited, in accordance with accepted academic practice. No use, distribution or reproduction is permitted which does not comply with these terms. 